\title{
OSTROWSKI TYPE INEQUALITIES
}

\author{
GEORGE A. ANASTASSIOU
}

(Communicated by Andrew M. Bruckner)

\begin{abstract}
Optimal upper bounds are given to the deviation of a function $f \in C^{N}([a, b]), N \in \mathbb{N}$, from its averages. These bounds are of the form $A \cdot\left\|f^{(N)}\right\|_{\infty}$, where $A$ is the smallest universal constant, i.e., the produced inequalities are sharp and sometimes are attained. This work has been greatly motivated by the works of Ostrowski (1938) and Fink (1992).
\end{abstract}

\section{INTRODUCTION}

Here we establish optimal upper bounds on the deviation of a function from its averages. These lead to sharp inequalities. Namely, let $f \in C^{n+1}([a, b])$, $n \in \mathbb{Z}_{+}$, such that $f^{(k)}(x)=0, k=1, \ldots, n$, where $x$ is a fixed point in $[a, b]$. Then we establish that

$$
\left|\frac{1}{b-a} \cdot \int_{a}^{b} f(y) d y-f(x)\right| \leq \varphi_{n}(x) \cdot\left\|f^{(n+1)}\right\|_{\infty},
$$

where $\varphi_{n}(x)$ is a continuous function that depends only on $n, a, b$, it has a simple form and it is the smallest possible, i.e., $(*)$ is sharp and in some cases it is even attained. The special case of $x=\frac{a+b}{2}$ is encountered.

\section{ON OSTROWSKI'S INEQUALITY}

Ostrowski's inequality (see Ostrowski [2]) is as follows:

$$
\left|\frac{1}{b-a} \cdot \int_{a}^{b} f(y) d y-f(x)\right| \leq\left(\frac{1}{4}+\frac{\left(x-\frac{a+b}{2}\right)^{2}}{(b-a)^{2}}\right) \cdot(b-a) \cdot\left\|f^{\prime}\right\|_{\infty},
$$

where $f \in C^{1}([a, b]), x \in[a, b]$. Inequality (1) is sharp since the function in ( ) cannot be replaced by a smaller one. One can easily notice that

$$
\left(\frac{1}{4}+\frac{\left(x-\frac{a+b}{2}\right)^{2}}{(b-a)^{2}}\right) \cdot(b-a)=\frac{(x-a)^{2}+(b-x)^{2}}{2 \cdot(b-a)} .
$$

Received by the editors October 13, 1993 and, in revised form, June 7, 1994.

1991 Mathematics Subject Classification. Primary 26D07, 26D10, 26D15, 41A44; Secondary 26A24, 26D20.

Key words and phrases. Function average, deviation of a function, best constant, sharp/attained inequality. 
Next, we give a different proof to (1) from that of Ostrowski's initial proof of 1938 in [2].

Theorem 1. Let $f \in C^{1}([a, b]), x \in[a, b]$. Then

$$
\left|\frac{1}{b-a} \cdot \int_{a}^{b} f(y) d y-f(x)\right| \leq\left(\frac{(x-a)^{2}+(b-x)^{2}}{2 \cdot(b-a)}\right) \cdot\left\|f^{\prime}\right\|_{\infty} .
$$

Inequality (3) is sharp, namely the optimal function is

$$
f^{*}(y):=|y-x|^{\alpha} \cdot(b-a), \quad \alpha>1 .
$$

Proof. Observe that

$$
\begin{aligned}
\left|\frac{1}{b-a} \cdot \int_{a}^{b} f(y) d y-f(x)\right| & =\frac{1}{(b-a)} \cdot\left|\int_{a}^{b}(f(y)-f(x)) d y\right| \\
& \leq \frac{1}{(b-a)} \cdot \int_{a}^{b}|f(y)-f(x)| \cdot d y \\
& \leq \frac{1}{(b-a)} \cdot\left\|f^{\prime}\right\|_{\infty} \cdot \int_{a}^{b}|y-x| \cdot d y \\
& =\frac{\left\|f^{\prime}\right\|_{\infty}}{2 \cdot(b-a)} \cdot\left((x-a)^{2}+(b-x)^{2}\right) .
\end{aligned}
$$

So, we have established inequality (3). Note that

$$
f^{* \prime}(y)=\alpha \cdot|y-x|^{\alpha-1} \cdot \operatorname{sign}(y-x) \cdot(b-a),
$$

thus

$$
\left|f^{* \prime}(y)\right|=\alpha \cdot|y-x|^{\alpha-1} \cdot(b-a)
$$

and

$$
\left\|f^{* \prime}\right\|_{\infty}=\alpha \cdot(b-a) \cdot(\max (b-x, x-a))^{\alpha-1} .
$$

Also we notice that $f^{*}(x)=0$.

Therefore we have for $f^{*}$ that

$$
\text { L.H.S.(3) }=\int_{a}^{b}|y-x|^{\alpha} \cdot d y=\frac{(x-a)^{\alpha+1}+(b-x)^{\alpha+1}}{\alpha+1}
$$

and

$$
\lim _{\alpha \rightarrow 1} \text { L.H.S. }(3)=\frac{(x-a)^{2}+(b-x)^{2}}{2} .
$$

Also, we observe that

$$
\text { R.H.S. }(3)=\left(\frac{(x-a)^{2}+(b-x)^{2}}{2}\right) \cdot \alpha \cdot(\max (b-x, x-a))^{\alpha-1}
$$

and

$$
\lim _{\alpha \rightarrow 1} \text { R.H.S. }(3)=\frac{(x-a)^{2}+(b-x)^{2}}{2},
$$

i.e.,

$$
\lim _{\alpha \rightarrow 1} \text { L.H.S.(3) }=\lim _{\alpha \rightarrow 1} \text { R.H.S.(3), }
$$

proving (3) sharp. 
Note that when $x=a$ or $x=b$, inequality (3) can be attained by $f_{a}(y):=$ $(y-a) \cdot(b-a), f_{b}(y):=(y-b) \cdot(b-a)$, respectively (then both sides of $(3)$ are equal to $\left.(b-a)^{2} / 2\right)$.

\section{MORE GENERAL OSTROWSKI TYPE INEQUALITIES}

The following material has been greatly motivated by the important work of Fink [1]. Let $f \in C^{n+1}([a, b]), n \in \mathbb{N}, x \in[a, b]$, be fixed. Then by Taylor's theorem we get

$$
f(y)-f(x)=\sum_{k=1}^{n} \frac{f^{(k)}(x)}{k !} \cdot(y-x)^{k}+\mathscr{R}_{n}(x, y),
$$

where

$$
\mathscr{R}_{n}(x, y):=\int_{x}^{y}\left(f^{(n)}(t)-f^{(n)}(x)\right) \cdot \frac{(y-t)^{n-1}}{(n-1) !} \cdot d t
$$

here $y$ can be $\geq x$ or $\leq x$.

Let $y \geq x$; then

$$
\begin{aligned}
\left|\mathscr{R}_{n}(x, y)\right| & \leq \int_{x}^{y}\left|f^{(n)}(t)-f^{(n)}(x)\right| \cdot \frac{(y-t)^{n-1}}{(n-1) !} \cdot d t \\
& \leq\left\|f^{(n+1)}\right\|_{\infty} \cdot \int_{x}^{y}|t-x| \cdot \frac{|y-t|^{n-1}}{(n-1) !} \cdot d t \\
& =\left\|f^{(n+1)}\right\|_{\infty} \cdot \frac{(y-x)^{n+1}}{(n+1) !}
\end{aligned}
$$

i.e.,

$$
\left|\mathscr{R}_{n}(x, y)\right| \leq \frac{\left\|f^{(n+1)}\right\|_{\infty}}{(n+1) !} \cdot(y-x)^{n+1}, \quad y \geq x
$$

Now let $x \geq y$; then

$$
\begin{aligned}
\left|\mathscr{R}_{n}(x, y)\right| & =\left|\int_{y}^{x}\left(f^{(n)}(t)-f^{(n)}(x)\right) \cdot \frac{(y-t)^{n-1}}{(n-1) !} \cdot d t\right| \\
& \leq \int_{y}^{x}\left|f^{(n)}(t)-f^{(n)}(x)\right| \cdot \frac{|y-t|^{n-1}}{(n-1) !} \cdot d t \\
& \leq \frac{\left\|f^{(n+1)}\right\|_{\infty}}{(n-1) !} \cdot \int_{y}^{x}(x-t) \cdot(t-y)^{n-1} \cdot d t \\
& =\frac{\left\|f^{(n+1)}\right\|_{\infty}}{(n+1) !} \cdot(x-y)^{n+1}
\end{aligned}
$$

i.e.,

$$
\left|\mathscr{R}_{n}(x, y)\right| \leq \frac{\left\|f^{(n+1)}\right\|_{\infty}}{(n+1) !} \cdot(x-y)^{n+1}, \quad x \geq y .
$$

From (8) and (9) we get

$$
\left|\mathscr{R}_{n}(x, y)\right| \leq \frac{\left\|f^{(n+1)}\right\|_{\infty}}{(n+1) !} \cdot|y-x|^{n+1}, \quad \text { for all } x, y \in[a, b] .
$$


Next we treat

$$
\begin{aligned}
& \left|\frac{1}{b-a} \cdot \int_{a}^{b} f(y) d y-f(x)\right|=\frac{1}{b-a} \cdot\left|\int_{a}^{b}(f(y)-f(x)) \cdot d y\right| \\
& =\frac{1}{b-a} \cdot\left|\int_{a}^{b}\left[\sum_{k=1}^{n} \frac{f^{(k)}(x)}{k !} \cdot(y-x)^{k}+\mathscr{R}_{n}(x, y)\right] \cdot d y\right| \\
& \quad=\frac{1}{b-a} \cdot\left|\sum_{k=1}^{n} \frac{f^{(k)}(x)}{k !} \cdot \int_{a}^{b}(y-x)^{k} \cdot d y+\int_{a}^{b} \mathscr{R}_{n}(x, y) \cdot d y\right| \\
& =\frac{1}{b-a} \cdot \mid \sum_{k=1}^{n} \frac{f^{(k)}(x)}{(k+1) !} \cdot\left[(b-x)^{k+1}-(a-x)^{k+1}\right] \\
& \leq \frac{1}{b-a} \cdot\left[\sum_{k=1}^{n} \frac{\left|f^{(k)}(x)\right|}{(k+1) !} \cdot\left|(b-x)^{k+1}-(a-x)^{k+1}\right|\right. \\
& \left.\quad+\frac{\left\|f^{(n+1)}\right\|_{\infty}}{(n+1) !} \cdot \int_{a}^{b}|y-x|^{n+1} \cdot d y\right]
\end{aligned}
$$

i.e., we have proved that

$$
\begin{aligned}
\left|\frac{1}{b-a} \cdot \int_{a}^{b} f(y) d y-f(x)\right| \\
\leq \frac{1}{b-a} \cdot\left[\sum_{k=1}^{n} \frac{\left|f^{(k)}(x)\right|}{(k+1) !} \cdot\left|(b-x)^{k+1}-(a-x)^{k+1}\right|\right. \\
\left.\quad+\frac{\left\|f^{(n+1)}\right\|_{\infty}}{(n+2) !} \cdot\left((x-a)^{n+2}+(b-x)^{n+2}\right)\right]
\end{aligned}
$$

where $f \in C^{n+1}([a, b]), n \in \mathbb{N}, x \in[a, b]$, is fixed.

If we choose $x=\frac{a+b}{2}$, then

$$
b-x=x-a=\frac{b-a}{2} .
$$

Thus

(12)

$$
\begin{aligned}
& \left|\frac{1}{b-a} \cdot \int_{a}^{b} f(y) d y-f\left(\frac{a+b}{2}\right)\right| \\
& \quad \leq \frac{1}{b-a} \cdot\left[\sum_{1 \leq k \text { even } \leq n} \frac{\left|f^{(k)}\left(\frac{a+b}{2}\right)\right|}{(k+1) !} \cdot \frac{(b-a)^{k+1}}{2^{k}}+\frac{\left\|f^{(n+1)}\right\|_{\infty}}{(n+2) !} \cdot \frac{(b-a)^{n+2}}{2^{n+1}}\right],
\end{aligned}
$$

where $f \in C^{n+1}([a, b]), n \in \mathbb{N}$.

The above considerations and the established inequalities (11) and (12) lead to the following results. 
Theorem 2. Let $f \in C^{n+1}([a, b]), n \in \mathbb{N}$ and $x \in[a, b]$ be fixed, such that $f^{(k)}(x)=0, k=1, \ldots, n$. Then

$$
\left|\frac{1}{b-a} \cdot \int_{a}^{b} f(y) d y-f(x)\right| \leq \frac{\left\|f^{(n+1)}\right\|_{\infty}}{(n+2) !} \cdot\left(\frac{(x-a)^{n+2}+(b-x)^{n+2}}{b-a}\right) .
$$

Inequality (13) is sharp. Namely, when $n$ is odd it is attained by $f^{*}(y):=$ $(y-x)^{n+1} \cdot(b-a)$, while when $n$ is even the optimal function is

$$
\tilde{f}(y):=|y-x|^{n+\alpha} \cdot(b-a), \quad \alpha>1 .
$$

Proof. Inequality (13) comes immediately from (11). Next we prove the sharpness of inequality (13).

When $n$ is odd: Notice that $f^{*(k)}(x)=0, k=0,1, \ldots, n$, and $f^{*(n+1)}(y)$ $=(n+1) ! \cdot(b-a)$. Hence

$$
\left\|f^{*(n+1)}\right\|_{\infty}=(n+1) ! \cdot(b-a)
$$

Plugging $f^{*}$ into (13) we get

$$
\text { L.H.S. }(13)=\frac{(b-x)^{n+2}+(x-a)^{n+2}}{n+2} \text {. }
$$

Also,

$$
\text { R.H.S. }(13)=\frac{(x-a)^{n+2}+(b-x)^{n+2}}{n+2} .
$$

From (14) and (15), when $n$ is odd, inequality (13) was proved to be sharp, in particular attained by $f^{*}$.

When $n$ is even: Notice that $\tilde{f}^{(k)}(x)=0, k=0,1, \ldots, n$, and

$$
\tilde{f}^{(n+1)}(y)=(n+\alpha)(n+\alpha-1) \cdots(\alpha+1) \cdot \alpha \cdot|y-x|^{\alpha-1} \cdot \operatorname{sign}(y-x) \cdot(b-a) .
$$

Hence

$$
\left|\tilde{f}^{(n+1)}(y)\right|=\left(\prod_{j=0}^{n}(n+\alpha-j)\right) \cdot|y-x|^{\alpha-1} \cdot(b-a)
$$

and

$$
\left\|\tilde{f}^{(n+1)}\right\|_{\infty}=\left(\prod_{j=0}^{n}(n+\alpha-j)\right) \cdot(\max (b-x, x-a))^{\alpha-1} \cdot(b-a) .
$$

Consequently we have

$$
\begin{aligned}
\text { R.H.S. }(13)= & \frac{\left(\prod_{j=0}^{n}(n+\alpha-j)\right) \cdot(\max (b-x, x-a))^{\alpha-1}}{(n+2) !} \\
& \cdot\left((x-a)^{n+2}+(b-x)^{n+2}\right), \quad \alpha>1 .
\end{aligned}
$$

Thus

$$
\lim _{\alpha-1} \text { R.H.S. }(13)=\frac{(x-a)^{n+2}+(b-x)^{n+2}}{n+2}
$$

and

$$
\text { L.H.S. }(13)=\frac{(x-a)^{n+\alpha+1}+(b-x)^{n+\alpha+1}}{n+\alpha+1} \text {. }
$$


Therefore

$$
\lim _{\alpha \rightarrow 1} \text { L.H.S. }(13)=\frac{(x-a)^{n+2}+(b-x)^{n+2}}{n+2} .
$$

From (16) and (17) we get that (13) is sharp also when $n$ is even.

Note that when $x=a$ or $x=b$ and $n$ is even, inequality (13) can be attained by $\tilde{f}_{a}(y):=(y-a)^{n+1} \cdot(b-a), \tilde{f}_{b}(y):=(y-b)^{n+1} \cdot(b-a)$, respectively (then both sides of (13) are equal to $\left.(b-a)^{n+2} / n+2\right)$. When $x=(a+b) / 2$, we have a case of special interest which is described next.

Theorem 3. Let $f \in C^{n+1}([a, b]), n \in \mathbb{N}$ such that $f^{(k)}((a+b) / 2)=0$, all $k$ even $\in\{1, \ldots, n\}$. Then

$$
\left|\frac{1}{b-a} \cdot \int_{a}^{b} f(y) d y-f\left(\frac{a+b}{2}\right)\right| \leq \frac{\left\|f^{(n+1)}\right\|_{\infty}}{(n+2) !} \cdot \frac{(b-a)^{n+1}}{2^{n+1}} .
$$

Inequality (18) is sharp. Namely, when $n$ is odd it is attained by $f^{*}(y):=$ $\left(y-\frac{a+b}{2}\right)^{n+1} \cdot(b-a)$, while when $n$ is even the optimal function is

$$
\tilde{f}(y):=\left|y-\frac{a+b}{2}\right|^{n+\alpha} \cdot(b-a), \quad \alpha>1 .
$$

Corollary 1. Let $f \in C^{2}([a, b])$ such that $f^{\prime \prime}((a+b) / 2)=0$. Then

$$
\left|\frac{1}{b-a} \cdot \int_{a}^{b} f(y) d y-f\left(\frac{a+b}{2}\right)\right| \leq\left\|f^{\prime \prime}\right\|_{\infty} \cdot \frac{(b-a)^{2}}{24},
$$

which is sharp as in Theorem 3.

Proof. Apply Theorem 3 with $n=1$.

Proof of Theorem 3. Inequality (18) comes immediately from (12) and the assumption $f^{(k)}((a+b) / 2)=0$, all $k$ even in $\{1, \ldots, n\}$.

Next we prove the sharpness of inequality (18).

When $n$ is odd: We notice that

$$
f^{*(k)}\left(\frac{a+b}{2}\right)=0, \quad \text { for } k=0 \text { and all } k \text { even } \in\{1, \ldots, n\},
$$

and furthermore

$$
f^{*(n+1)}(y)=(n+1) ! \cdot(b-a), \quad \text { all } y \in[a, b] .
$$

Thus

$$
\text { R.H.S. }(18)=\frac{(b-a)^{n+2}}{(n+2) \cdot 2^{n+1}}
$$

Also we find

$$
\text { L.H.S. }(18)=\frac{(b-a)^{n+2}}{(n+2) \cdot 2^{n+1}} .
$$

From (20) and (21) we get that (18) is attained by $f^{*}$, therefore (18) has been proved as sharp when $n$ is odd. 
When $n$ is even: We notice that furthermore $\tilde{f}^{(k)}((a+b) / 2)=0$, for $k=0$ and all $k$ even in $\{1, \ldots, n\}$,

$$
\tilde{f}^{(n+1)}(y)=\prod_{j=0}^{n}(n+\alpha-j) \cdot\left|y-\frac{a+b}{2}\right|^{\alpha-1} \cdot \operatorname{sign}\left(y-\frac{a+b}{2}\right) \cdot(b-a)
$$

and

$$
\left\|\tilde{f}^{(n+1)}\right\|_{\infty}=\left(\prod_{j=0}^{n}(n+\alpha-j)\right) \cdot\left(\frac{b-a}{2}\right)^{\alpha-1} \cdot(b-a) .
$$

Thus

$$
\begin{aligned}
\text { R.H.S.(18) }= & \frac{\left(\prod_{j=0}^{n}(n+\alpha-j)\right) \cdot((b-a) / 2)^{\alpha-1} \cdot(b-a)}{(n+2) !} \\
& \cdot \frac{(b-a)^{n+1}}{2^{n+1}}, \quad \alpha>1 .
\end{aligned}
$$

Hence

$$
\lim _{\alpha \rightarrow 1} \text { R.H.S. }(18)=\frac{(b-a)^{n+2}}{(n+2) \cdot 2^{n+1}}
$$

Also we find

and

$$
\text { L.H.S. }(18)=\frac{2 \cdot((b-a) / 2)^{n+\alpha+1}}{n+\alpha+1}
$$

From (22) and (23) we have established that inequality (18) is sharp again when $n$ is even.

\section{REFERENCES}

1. A. M. Fink, Bounds on the deviation of a function from its averages, Czechoslovak Math. J. 42 (117) (1992), 289-310.

2. A. Ostrowski, Über die Absolutabweichung einer differentiebaren Funktion von ihrem Integralmittelwert, Comment. Math. Helv. 10 (1938), 226-227.

Department of Mathematical Sciences, The University of Memphis, Memphis, TenNESSEE 38152 